\author{
Revista Calidad en la Educación Superior \\ Programa de Autoevaluación Académica \\ Universidad Estatal a Distancia, Costa Rica \\ ISSN 1659-4703 \\ revistacalidad@uned.ac.cr
}

\title{
EL EFECTO DE LA CALIDAD EN UN PROGRAMA DE ESTUDIO ACREDITADOS: CASO DEL BACHILLERATO EN LA ENSEÑANZA DEL INGLÉS EN LA UNIVERSIDAD NACIONAL, COSTA RICA
}

\author{
THE EFFECT OF THE QUALITY OF AN ACCREDITATED STUDIES PROGRAM: \\ THE CASE OF THE BACHELOR IN ENGLISH TEACHING \\ AT UNIVERSIDAD NACIONAL, COSTA RICA
}

\section{Lindsay A. Chaves Fernández ${ }^{1}$ lindsay.chaves.fernandez@una.cr Didier Rojas Cerdas ${ }^{2}$ didierrojas921@hotmail.com Olga L. Chaves Carballo 3 profeolgachaves@hotmail.com Escuela de Literatura y Ciencias del Lenguaje, Universidad Nacional de Costa Rica}

"La Educación no cambia al mundo: cambia a las personas que van a cambiar el mundo." Paulo Freire

\author{
Volumen 6, Número 2 \\ Noviembre 2015 \\ Pp. $179-199$
}

Recibido: 16 de agosto, 2015

Aprobado: 28 de setiembre, 2015

\footnotetext{
${ }^{1}$ M.A. Lindsay Chaves Fernández tiene una Maestría en Segundas Lenguas y Culturas con Énfasis en Alumnado Adulto y un Bachillerato en la Enseñanza del Inglés de la Universidad Nacional de Costa Rica. Es profesora de la Escuela de Literatura y Ciencias del Lenguaje en la UNA desde el año 2012.

${ }^{2}$ M.A. Didier Rojas Cerdas tiene una Maestría en Segundas Lenguas y Culturas con Énfasis en Alumnado Adulto de la UNA y un Bachillerato en la Enseñanza del Inglés de la UCR. Trabaja en la Escuela de Literatura y Ciencias del Lenguaje en la UNA desde el año 2008.

${ }^{3}$ Dra. Olga Chaves Carballo tiene un doctorado en Educación con Énfasis en Mediación Pedagógica de la Universidad de la Salle, y una Maestría en la Enseñanza del Inglés como Segundo Idioma y Administración educativa de la Universidad del Sur de Illinois, Estados Unidos. Es profesora-investigadora de la Escuela de Literatura y Ciencias del Lenguaje en la UNA desde el año 2003.
} 


\title{
Resumen
}

La Escuela de Literatura y Ciencias del Lenguaje de la Universidad Nacional, Costa Rica posee un Bachillerato en la Enseñanza del Inglés, acreditado por SINAES desde el año 2006. Este artículo presenta una investigación acerca del alcance de esta carrera en la preparación adecuada de sus estudiantes. Para realizar esta investigación, los estudiantes de primer ingreso, así como los de cuarto año realizaron una encuesta sobre la acreditación y el plan de estudios, respectivamente. Los resultados muestran que más del $94 \%$ de los estudiantes están informados sobre la acreditación, y manifiestan satisfacción con las competencias pedagógicas y lingüísticas adquiridas en su plan de estudios. Los investigadores concluyen que este bachillerato está cumpliendo con los objetivos de mejora permanente que implica tener una carrera acreditada.

Palabras clave: Enseñanza del inglés, calidad, acreditación, programa de estudios, profesores de inglés.

\begin{abstract}
The School of Literature and Languages of the Universidad Nacional de Costa Rica has a Bachelor's Degree in English Teaching, accredited by SINAES since the year 2006. This article presents a research about the scope of this major on the students' adequate education. Freshmen students of the major answered a survey related to their awareness regarding the accreditation, and senior students answered a questionnaire about the quality of the major's curriculum. The results show that over $94 \%$ of the students are informed about the accreditation, and express satisfactory feelings about the pedagogical and linguistic competencies acquired in the major. The researchers conclude that this degree is accomplishing the objectives of the ongoing improvement required by SINAES accreditation.
\end{abstract}

Keywords: English teaching, quality, accreditation, curriculum, English teachers.

\section{Introducción}

Un programa de estudio acreditado por el Sistema Nacional de Acreditación de la Educación Superior (SINAES), garantiza que los estudiantes se gradúen de carreras con alto nivel de calidad, ya que al estar acreditadas se logra a cabalidad el cumplimiento de los requerimientos ineludibles para su formación profesional. La sociedad costarricense necesita graduados en el área de inglés con un nivel de lengua avanzado y las estrategias pedagógicas necesarias que le permitan desempeñarse exitosamente en su campo laboral. 
El Bachillerato en la Enseñanza del Inglés (BEI) enmarca una estrategia de mejoramiento continuo de la carrera, que establece un círculo de calidad basado en la autoevaluación, la evaluación externa, la acreditación, el mejoramiento permanente y la documentación. En el año 2001, la Escuela de Literatura y Ciencias del Lenguaje (ELCL) acordó iniciar el proceso con miras a la acreditación del BEI (Acta de Asamblea de Unidad Académica, sesión ordinaria $\left.\mathrm{N}^{\circ} 02-2001\right)$. Los académicos del área de inglés acordaron someter el $\mathrm{BEI}$ al proceso de autoevaluación y como resultado, después de un riguroso proceso de autoanálisis y de visita de evaluadores externos, el BEI es acreditado en mayo de 2006 por un período de 4 años (Oficio SINAES-236-2006). Posteriormente, en el año 2011, se logra reacreditar la carrera por un período de 6 años más (Acuerdo del SINAES, Sesión 692, 14 de octubre de 2011). En enero de 2014 inicia la tercera etapa, con el proyecto titulado Gestión de la Calidad del BEI, cuyo propósito es mantener los estándares de calidad para ofrecer a la comunidad nacional una carrera de alto nivel en la formación de docentes en la enseñanza del inglés. El proceso de autoevaluación y acreditación contribuye con el mejoramiento de la gestión, y por consiguiente, la calidad de los servicios que ofrecen las instituciones de educación superior con el fin de que los estudiantes gocen de las mejores condiciones académicas. A la luz de este enunciado, este artículo tiene como propósito investigar la calidad que ostenta esta carrera acreditada en la formación académica de los estudiantes y la preparación en su futura labor profesional, cuyo fin social es educar a los futuros estudiantes de secundaria y de otros sectores tanto públicos como privados.

\section{Contextualización}

Una carrera acreditada garantiza la calidad de un plan de estudios para proveer a la sociedad de profesionales de alto nivel en su área. En la Escuela de Literatura y Ciencias del Lenguaje de la Facultad de Filosofía y Letras en la Universidad Nacional (UNA), el compromiso de mantener la calidad de la educación nos lleva a fortalecer cada componente de la carrera, y así ofrecer a los estudiantes las mejores condiciones curriculares para su formación profesional, lo cual lo destacará en su campo laboral. 
Cabe destacar que la Ley de Fortalecimiento del SINAES ( $\left.N^{\circ} 8798,2010\right)$, dispone que "los criterios y estándares definidos por el SINAES tendrán carácter oficial de norma académica nacional de calidad". Además, el artículo 4 de la Ley 8798 establece que "El estado y sus instituciones procurarán contratar preferentemente al personal graduado de carreras oficialmente acreditadas". Igualmente, el Plan Estratégico de Mediano Plazo (2013-2017) considera "las acciones tendientes a renovar nuestra actividad académica y a adaptarla creativamente a los cambios constantes que se generan en la sociedad contemporánea, requiere graduados con perfiles interdisciplinarios y versátiles" (p.11).

Los expertos en gestión de la calidad de la educación superior concuerdan que la calidad en la formación de los principales actores, los estudiantes, depende de factores tales como profesores de alta calidad, un plan de estudios no sólo actualizado sino pertinente a las demandas sociales y profesionales, recursos, e infraestructura. Barragán (2009) resalta que los resultados de las instituciones académicas, o sea, los egresados, los contenidos de los planes y los programas son los que materializan la calidad de la educación. Igualmente, Mora y León (2012) manifiestan al respecto:

La importancia creciente de los procesos de aseguramiento de la calidad en la educación superior de América Latina, en el marco de los cambios experimentados en los Sistemas de Educación Superior (SES), durante las últimas cuatro décadas, se relacionan de manera estrecha con las nuevas demandas originadas en la sociedad, la creciente integración del conocimiento, los sistemas educativos y la cultura, así como con la persistencia de notables debilidades en el funcionamiento de las instituciones de educación superior (IES) y en los programas o carreras llevadas a cabo en las IES de la región. Estas debilidades limitan las posibilidades de atender, de forma eficaz, a las demandas de la sociedad y a las exigencias de su integración en la sociedad del conocimiento (p. 16).

Precisamente, estas son algunas de las razones que justifican las acciones de mejoramiento y de sostenibilidad de la calidad de un programa de estudio acreditado por una entidad de trascendencia internacional como lo es el SINAES. 


\section{La importancia del inglés en nuestro país}

Desde hace décadas el inglés se ha convertido en un pilar esencial para el desarrollo económico y social del país. Iniciativas, como el programa Costa Rica Bilingüe, han impulsado el aprendizaje del inglés permitiendo mejorar las oportunidades laborales de aquellos profesionales que hablan inglés, según decreto № 34425-MEP-COMEX del 11 de marzo de 2008, cuyo objetivo es dotar a la comunidad nacional de las competencias lingüísticas que le permitan aumentar su competitividad. Esta iniciativa ha servido como estrategia de desarrollo para el país, dado que propicia, no solo el mejoramiento de las condiciones laborales de los individuos sino que es, a su vez, una ventana de oportunidades para jóvenes y adultos hacia el aprendizaje de idiomas extranjeros con un enfoque específico según las necesidades del mercado global en el cual están inmersos (Presidencia de la República, 2008-2017). Tal y como lo describen Bassi y Álvarez (2015),

Dominar el inglés como segunda lengua será (y es) una habilidad indispensable para acceder a mejores oportunidades en este nuevo siglo. La lengua inglesa es la más útil para la comunicación internacional, interregional y multilateral y es la lengua franca en ámbitos como el mercadeo, el comercio internacional y el turismo (s.p.).

Este análisis de la realidad revela la necesidad de graduar más profesionales universitarios con un alto nivel en su campo de estudio, pues ellos son quienes contribuirán con el desarrollo económico, social y educativo del país. Dentro de los esfuerzos realizados en Costa Rica, existe el convenio con la compañía multinacional SYKES, la cual desde el año 2007 tiene como objetivo incrementar las habilidades lingüísticas en inglés de muchas personas. Se estima que más de 5000 personas han sido reclutadas por esta empresa y que 4000 se han graduado de este convenio. SYKES ha brindado apoyo al sistema educativo nacional en el mejoramiento del inglés, así como en el desarrollo técnico e inclusión laboral de la juventud costarricense. Para el Ministerio de Trabajo la firma de este convenio significó un gran avance en los esfuerzos que realizan la empresa privada y el gobierno en la búsqueda de brindar más y mejores opciones de empleo para los costarricenses (Gobierno de la República, 2015). 
Además, en cuanto a la pregunta: ¿Qué tan preparados están los costarricenses en el uso del inglés?, Fernández (2015) registra que Costa Rica tiene el primer lugar en cuanto a las notas en Latinoamérica en la prueba de manejo del inglés como segunda lengua. Estos datos aseguran que nuestro país está avanzando en el objetivo de lograr que los habitantes tengan un alto dominio del inglés.

En general, el país ha tomado una buena ruta con acciones claras y directas para dar respuesta a una necesidad que aclama un país con profesionales bilingües para contribuir al fenómeno de la globalización, la comunicación, la adquisición de conocimiento e información, y en el campo laboral.

\section{La formación de los estudiantes del Bachillerato en la Enseñanza del Inglés en la UNA}

El plan de estudio del BEI en la UNA se mantiene en los más altos niveles de calidad en todos sus componentes para asegurar que los estudiantes adquieran el nivel lingüístico y pedagógico requerido por las demandas sociales del país. Esto se comprueba con los estándares de calidad alcanzados por medio de la acreditación desde el año 2006 y reacreditada hasta el año 2017. Como se describe en el rediseño del Plan de Estudio (2013, p. 3), el principal objetivo del BEI es

Contribuir a la transformación de la sociedad costarricense hacia un desarrollo integral, autónomo y sostenible, formando profesionales críticos, independientes, participativos y creativos, con un dominio sólido del conocimiento y su problemática pedagógica, y formar integralmente educadores para la enseñanza del inglés, desde un currículum interdisciplinario que articule la educación con otros campos del conocimiento y fortalezca la identidad profesional de los docentes.

Por lo tanto, un proceso de acreditación nos orienta a la práctica reflexiva y a tomar en cuenta las demandas sociales al diseñar los programas de estudio. De esta manera, garantizamos que los estudiantes se formen para enfrentar diferentes realidades educativas. Los procesos de autoevaluación y acreditación han orientado los esfuerzos para formar 
estudiantes con una visión holística de la educación y capaces de mejorar las realidades sociales del país.

Para tal fin, el Área de Inglés del BEl aprobó, el 5 de agosto de 2014, las características deseables en el aspirante al ingresar a esta carrera, las cuales se encuentran en el catálogo titulado Información General Plan de Estudios (2014). Estas características se detallan a continuación (p. 20):

Es recomendable que los aspirantes a ingresar al Bachillerato en la Enseñanza del Inglés de la Escuela de Literatura y Ciencias del Lenguaje tengan interés, curiosidad e iniciativa por:

- Aprender una lengua extranjera;

- leer con propósitos académicos y de entretenimiento, lo cual permitirá el manejo de la amplia bibliografía existente en la lengua, así como la adquisición de vocabulario y estructuras gramaticales;

- investigar, utilizando para ello múltiples fuentes bibliográficas impresas y en línea;

- manejar tecnologías de comunicación e informática y tener disposición para aprender el uso de nuevas tecnologías;

- trabajar de manera individual y en equipo;

- compartir ideas y opiniones;

- interactuar con personas de otros países y otras culturas;

- organizar y planificar actividades;

- desarrollar destrezas sociales, empatía, sensibilidad y respeto hacia todas las formas de diversidad;

- desarrollar la vocación y las aptitudes para ejercer la enseñanza del inglés a adolescentes y adultos. 
Además, dentro del Plan de Estudios (Rediseño del BEI 2013, p. 52-193), en cada curso, los estudiantes desarrollan paulatinamente las siguientes habilidades:

- Revisión y análisis bibliográfico;

- análisis de las estructuras del idioma inglés y de las obras literarias escritas en inglés;

- realización de trabajos de investigación en las bibliotecas y en comunidades e instituciones;

- presentaciones orales sobre diversos temas y participación en debates;

- participación en grupos de trabajo y diálogos;

- realización de ensayos sobre diferentes temas;

- elaboración de pruebas y de material didáctico;

- cumplimiento de la práctica docente.

Los objetivos del Plan de Estudios (2013, p. 1-2) estipulan que los estudiantes graduados del $\mathrm{BEI}$ son "profesionales críticos, independientes, participativos y creativos con un dominio sólido del conocimiento y su problemática pedagógica". Asimismo, se forman "educadores integrales para la enseñanza, desde un currículum interdisciplinario que articule la educación con otros campos del conocimiento y fortalezca la identidad profesional de los docentes". Además, el sentido de pertenencia cultural con principios y valores democráticos y la vocación investigadora son componentes esenciales dentro de la formación profesional de los estudiantes.

\section{Metodología de este estudio}

La primera acción para realizar esta investigación fue el diseño de una encuesta en línea con la herramienta LimeSurvey para identificar el nivel de información de los estudiantes de nuevo ingreso acerca del plan de estudio acreditado con que cuenta el BEI al que ingresaron. Esta encuesta fue contestada por un grupo de 39 estudiantes de primer ingreso, en el año 2014, en los meses de abril y mayo. Los resultados revelan que, en cuanto a la acreditación, los estudiantes tienen amplia información y la consideran de suma importancia. Las siguientes tablas demuestran las opiniones de los jóvenes encuestados: 
Tabla 1

Conocimiento de los estudiantes sobre BEI acreditado por el SINAES

\begin{tabular}{|l|c|c|}
\hline Aspecto evaluado & Cantidad & Porcentaje \\
\hline $\mathrm{Si}$ & 37 & $94.87 \%$ \\
\hline No & 2 & $5.13 \%$ \\
\hline
\end{tabular}

Fuente: Cuestionario aplicado a estudiantes de 1er nivel, Comisión Mejoramiento, UNA, abril-mayo, 2014

Tabla 2

Conocimiento de los estudiantes sobre el Plan de estudio del BEI

\begin{tabular}{|l|c|c|}
\hline Aspecto evaluado & Cantidad & Porcentaje \\
\hline Sí & 37 & $94.87 \%$ \\
\hline No & 2 & $5.13 \%$ \\
\hline
\end{tabular}

Fuente: Cuestionario aplicado a estudiantes de 1er nivel, Comisión Mejoramiento, UNA, abril-mayo, 2014

Tabla 3

Motivos que lo motivaron a ingresar a la carrera en el 2014

\begin{tabular}{|l|c|}
\hline \multicolumn{1}{|c|}{ Aspecto evaluado } & Cantidad \\
\hline Por interés en el área de estudio & 37 \\
\hline Por ser una carrera acreditada & 24 \\
\hline Por el prestigio de la UNA & 21 \\
\hline Por ser una opción para obtener trabajo & 12 \\
\hline
\end{tabular}

Fuente: Cuestionario aplicado a estudiantes de 1er nivel, Comisión Mejoramiento, UNA, abril-mayo, 2014

Mediante la información en las tablas anteriores se evidencia no sólo el conocimiento con que cuentan los estudiantes de primer ingreso acerca de la acreditación, sino que es posible palpar que este conocimiento influyó en su decisión de ingresar a una carrera acreditada en una institución de tan alto prestigio como lo es la UNA.

La segunda acción realizada por los investigadores fue el diseño de un cuestionario de 10 preguntas, tanto abiertas como cerradas, para valorar las impresiones de los estudiantes de cuarto año de la carrera, acerca de la calidad del plan de estudio (Anexo 1). Veintiún estudiantes participaron respondiendo este cuestionario, sus edades en un $90 \%$ oscilan entre los 20 y los 30 años y $10 \%$ con edades superiores a los 31 años de edad. El 
cuestionario se administró a los estudiantes en una de sus clases en el mes de junio de 2015. Los educandos opinan que en el plan de estudio de una carrera de enseñanza, las metodologías, las estrategias y las técnicas para enseñar una segunda lengua son herramientas vitales con las que se debe equipar a los aprendientes. Exitosamente, un $100 \%$ de los estudiantes opinan que éstas son estimuladas en el BEI acreditado. Los alumnos atribuyen su éxito académico a varios componentes trascendentales contemplados en toda carrera que haya pasado por el proceso de acreditación. Éstos se ven reflejados en la figura a continuación:

Figura 1

Factores que contribuyen al éxito académico

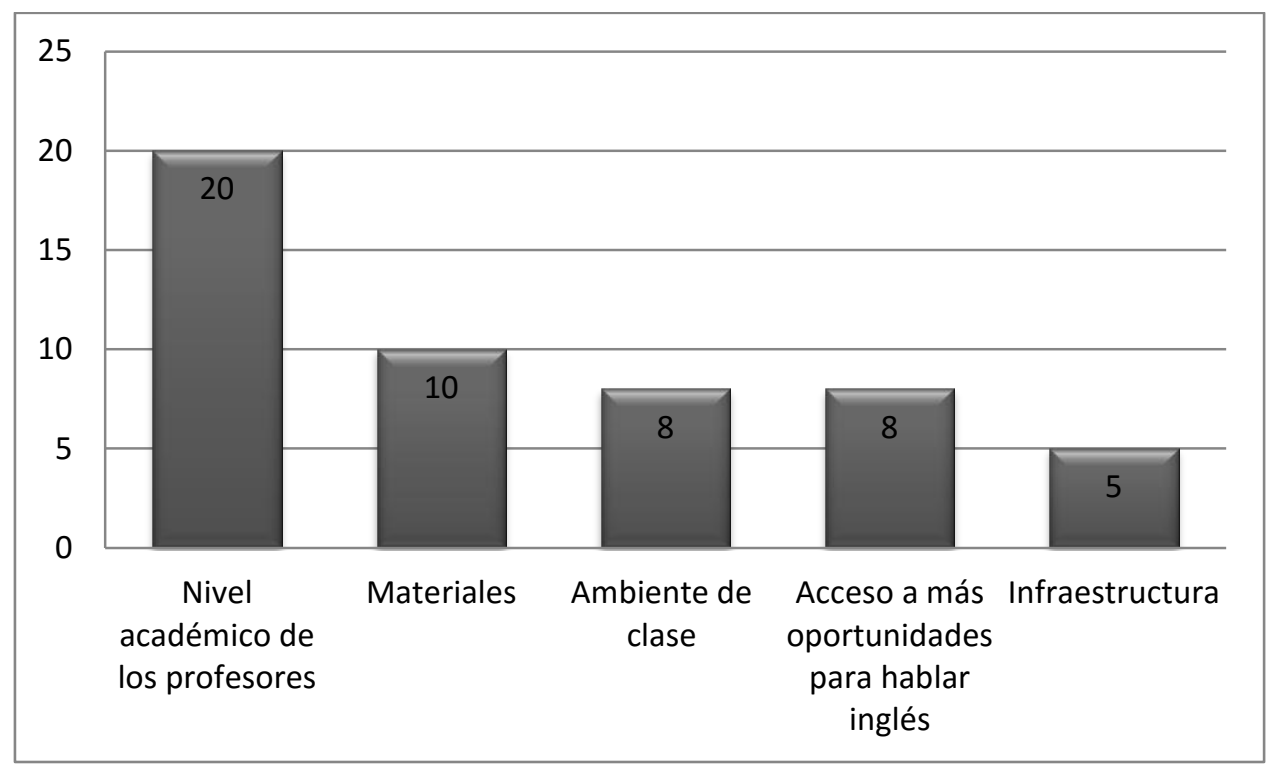

Fuente: Cuestionario aplicado a estudiantes de 4to nivel, Comisión Mejoramiento, UNA, junio 2015

Como es posible observar en la Figura 1, haber contado con profesores de alto nivel académico durante sus cuatro años de vida universitaria es de capital importancia para los estudiantes, ya que de esta manera pueden desarrollar una mayor competencia lingüística y pedagógica. Además del alto nivel académico de sus profesores, haber contado con los recursos necesarios durante sus cuatro años de estudio, haber tenido el ambiente propicio para el desarrollo de sus competencias, y haber tenido acceso a mayores oportunidades de interactuar con nativos parlantes de la lengua meta hace que los estudiantes se sientan 
seguros de contar con mayores oportunidades a nivel laboral por el reconocimiento y prestigio que ofrece una carrera acreditada de la UNA.

Asimismo, los alumnos consideran que, durante sus años de estudio, deben adquirir destrezas substanciales para un desarrollo adecuado de sus potencialidades como futuros profesores de inglés. Estas destrezas y su importancia se observan en la Figura 2 que se detalla a continuación:

\section{Figura 2}

\section{Destrezas más importantes a desarrollar}

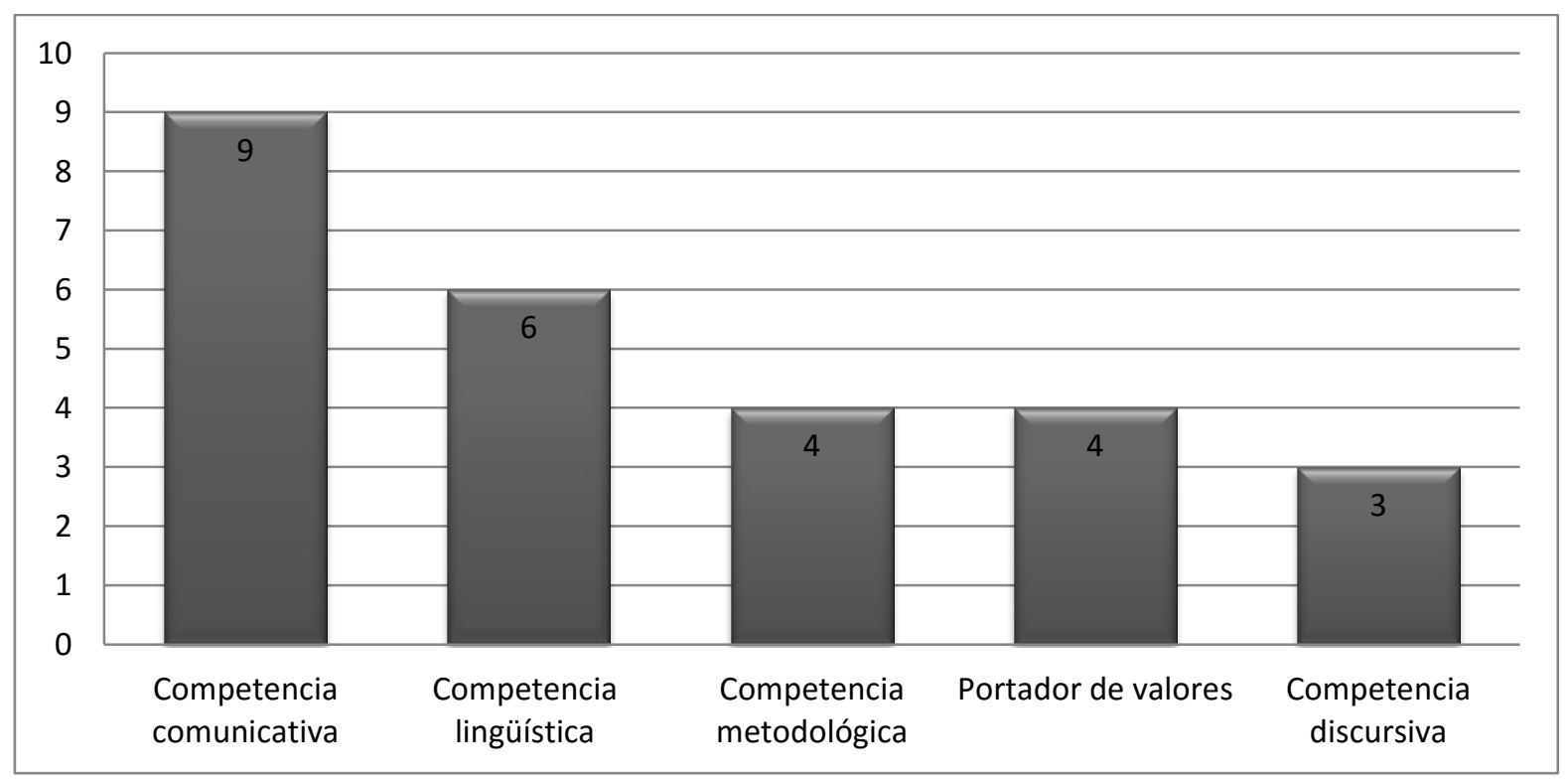

Fuente: Cuestionario aplicado a estudiantes de 4to nivel, Comisión Mejoramiento, UNA, junio 2015.

Los aprendientes encuestados expresaron sentirse satisfechos en cuanto al nivel de dominio de las competencias anteriormente ilustradas. Al mismo tiempo, opinan que gracias a la carrera acreditada cuentan con las competencias pedagógicas, lingüísticas y culturales para desenvolverse como profesionales en un futuro muy cercano. Ellos expresan que "[la carrera acreditada] prepara a los estudiantes con un nivel de inglés excelente, posee un plan enfocado en comunicación, y los profesores tienen las capacidades necesarias para enseñar los temas" (Opinión de estudiante de 4to año en encuesta realizada por los investigadores). 
Conjuntamente, otro factor valioso para los alumnos es, sin duda alguna, las futuras oportunidades laborales que se les presentarán al ingresar al mercado laboral después de su graduación. Al respecto, los estudiantes expresan sentirse muy confiados, ya que al poseer un título universitario acreditado están seguros de las posibilidades para conseguir un empleo porque "el título al ser acreditado tiene peso extra y una mayor consideración [por los empleadores]" (Opinión de estudiante de 4to año en encuesta realizada por los investigadores). Simultáneamente, los alumnos opinan que "[las oportunidades laborales] son muy buenas, ya que hoy en día el inglés es necesario para muchos campos, por lo que los profesores son muy buscados [por los empleadores]" (Opinión de estudiante de 4to año en encuesta realizada por los investigadores).

Por otra parte, los investigadores consideramos muy valioso considerar la motivación con la que cuentan los futuros docentes en la enseñanza del inglés, ya que una sociedad con profesionales motivados es en definitiva una sociedad con grandes posibilidades de desarrollar individuos integrales y plenos. Nias (1999) señala, "los profesores, al percibir su trabajo como vocación, llamada o misión, son conscientes de que éste tiene una dimensión ética. Un valor central para ellos es la idea de que son moralmente más que legalmente responsables de sus alumnos y experimentan un deseo de cuidar de ellos".

Al cuestionar a los aprendientes acerca de la inmediata contribución a la educación costarricense al integrarse al campo laboral de nuestro país, ellos respondieron con ideas que demuestran el potencial alcanzado hasta el momento en sus años universitarios, y que dejan a la vista la pasión con la que asumirán el reto de ser educadores. Martínez (2011) expresa, "el ser humano es un suprasistema altamente complejo, pero más o menos integrado. El nivel de integración armónica determina el grado de desarrollo y madurez de su personalidad. Todo esto impone a la educación una tarea o misión sumamente ardua y difícil". Sin embargo, cuando los profesionales están preparados para asumir este reto, la tarea ardua y difícil se vuelve un desafío que se asume con positivismo, buscando siempre una salida satisfactoria para todas las partes involucradas. Algunas de las acciones que los 
estudiantes de cuarto año del BEI acreditado esperan realizar al ejercer su profesión son (Opiniones de los estudiantes de 4to año en encuesta realizada por los investigadores):

- Desarrollar mis clases con énfasis en el pensamiento crítico;

- impulsar a los estudiantes a aprender el idioma para comunicarse en distintos contextos interculturales para interactuar con personas de diferentes contextos;

- proveer información que fomente el cambio de percepciones para mejorar la sociedad;

- promover valores;

- desarrollar clases constructivas;

- apoyar a los estudiantes, no solo académicamente, sino que comprender la situación de cada uno;

- motivar a los estudiantes al cambio en el aprendizaje;

- crear un ambiente participativo;

- ir más allá de un libro o lecciones impuestas por el MEP;

- desarrollar el pensamiento crítico y explotar su creatividad;

- influir positivamente en la percepción que tienen los estudiantes del inglés;

- ser un profesor diferente;

- preparar muchas actividades para no depender de un libro;

- incluir temas en contexto real, en el cual los estudiantes sean capaces de internalizar la lengua en vez de memorizar;

- lograr que aprendan el idioma de forma divertida y significativa.

Claramente, los futuros profesores de inglés se están comprometiendo, no sólo con el aprendizaje de una segunda lengua sino con su propio desarrollo, para llegar a ser y ayudar a desarrollar individuos integrales.

Adicionalmente, para este estudio, se analizaron los resultados obtenidos por los estudiantes en el Test of English for International Communication, TOEIC, el cual es un examen de inglés para profesionales que no tienen este idioma como lengua materna y mide las 
capacidades y competencias con que estos profesionales cuentan en su entorno laboral. Esta prueba contempla los lineamientos estipulados en el Marco Común Europeo de Referencia para los idiomas, el cual ha determinado seis niveles de competencia en el idioma inglés para establecer sistemas de capacidades de nivel lingüísticos de los estudiantes de lengua extranjera. Estos niveles de competencia son: A1, A2, B1, B2, C1 y C2; representando el A1 el nivel de competencia más básico en el idioma meta y el C2 el nivel de competencia de un nativo parlante del inglés.

En el año 2013, de 26 estudiantes, 13 se certificaron en el nivel C1, 11 en B2 y 1 en B1. En el 2014, 15 estudiantes tomaron el examen y de ellos 13 se certificaron con un C1 y 2 con B2. Estos resultados indican que los estudiantes se gradúan con el nivel correspondiente y deseado en un profesor de inglés requerido para trabajar en el MEP.

En suma, la acreditación del BEI es responsable de una serie de acciones de mejoramiento que han beneficiado a los estudiantes de diversas maneras, acciones que trascienden el quehacer de las Unidades Académicas responsables de la Carrera. El desarrollo de una cultura dirigida a la calidad ha permitido promover una gestión orientada a las necesidades e intereses del estudiantado, que es, al fin y al cabo, el principal beneficiario.

\section{Conclusiones}

En resumen, la carrera de Bachillerato en la Enseñanza del Inglés se caracteriza por su compromiso con el mejoramiento permanente para superar las debilidades detectadas en los procesos de autoevaluación y evaluación continua. Asimismo, se ha trabajado arduamente con el fin de darle sostenibilidad a las fortalezas identificadas en dichos procesos. Álvaro Cedeño Gómez (6 mayo, 2015), presidente del SINAES, señala que una carrera acreditada cuenta con beneficios tales como un programa de estudio revisado, docentes comprometidos, fomentación del pensamiento crítico, conexión con la sociedad, reconocimiento del Servicio Civil en puntuación ventajosa sobre estudiantes que no tienen una carrera acreditada. El estado global del BEI demuestra, sin lugar a duda, que el BEI es una carrera con alto nivel de calidad, comprometida con el mejoramiento continuo para dar 
respuesta a las necesidades educativas de la sociedad costarricense. Tal y como los estudiantes lo reflejaron en sus respuestas a las encuestas realizadas, el plan de estudio los forma de una manera holística y los prepara para asumir los retos educativos como futuros profesionales en su campo.

El principal objetivo de este proceso ha sido consolidar el compromiso de mejora permanente de la calidad de la carrera en todos sus aspectos, mediante la implementación de mecanismos para consolidar las fortalezas y acciones concretas. De este modo, la carrera espera seguir contribuyendo adecuadamente al desarrollo y progreso de la sociedad costarricense, mediante la formación de profesionales calificados. Sonia Mora Escalante, expresidenta del SINAES (30 julio, 2013) señala muy acertadamente que el SINAES es un sello que garantiza calidad a nivel nacional e internacional y esto a su vez se concreta en una mayor valoración de instituciones públicas para aquellos estudiantes que cursen sus estudios en una carrera acreditada.

\section{Referencias}

Acuerdo del SINAES, Sesión 692, 14 de octubre de 2011.

Barboza, G. (2013). La Educación Pública en Costa Rica: la enseñanza y el aprendizaje del idioma inglés. Recuperado de http://www.englishincostarica.org/maeugenia1.html.

Barragán, J. (2009). Estándares característicos de una escuela internacional: el impacto de la acreditación internacional de programas en la educación superior. Recuperado de http://www.spentamexico.org/v4-n2/4\%282\%29\%20174-187.pdf.

Bassi, M. y Álvarez, H. (2015). Habilidades para el Siglo XXI, La Enseñanza del Inglés en Costa Rica. Recuperado de http://www.iadb.org/es/temas/educacion/habilidades-parael-siglo-xxi-la-ensenanza-del-ingles-en-costa-rica,6641.html.

Cedeño, A. (2015). Beneficios de graduarse de una carrera acreditada.[Archivo de video]. Recuperado de https://www.youtube.com/watch?v=Ta95ZWpMmSE.

EF English Proficiency Index for Companies. (s.f.). Recuperado de http://www.ef.com.es/epi/epi-c. 
Fernández, A. (2015). Costa Rica: el mejor de América Latina en Prueba Global de Inglés (TOEFL). Recuperado de http://www.elfinancierocr.com/economia-y-politica/CostaRica-America-Latina-ingles_0_752324765.html.

Gobierno de la República. (2015). Mejor inglés para costarricenses gracias a convenio con SYKES. Recuperado de http://presidencia.go.cr/sin-categoria/mejor-ingles-paracostarricenses-gracias-a-convenio-con-sykes.

Hernández, A. (2008). El Inglés en Costa Rica: requisito Indispensable en un Mundo Globalizado. Actualidades Investigativas en Educación, 8, 23. Recuperado de http://revista.inie.ucr.ac.cr/autores/controlador/Article/accion/show/articulo/el-inglesen-costa-rica-requisito-indispensable-en-un-mundo-globalizado.html.

Información General Plan de Estudios. Bachillerato en la Enseñanza del Inglés. (2014). Facultad de Filosofía y Letras. Escuela de Literatura y Ciencias del Lenguaje. Universidad Nacional, Heredia, Costa Rica.

Lorenzana, R . (2012). La evaluación de los aprendizajes basada en competencias en la enseñanza universitaria . Recuperado de http://d-nb.info/1029421889/34.

Martínez, M. (2011). Dimensiones básicas de un desarrollo humano Integral. Recuperado de http://polis.revues.org/1802.

Mora, J. y León G. (2012). Efectos de la acreditación en carreras universitarias seleccionadas en la educación universitaria de C.R. La Nación. Cuarto informe del estado de la educación.

Mora, S. (2013). Beneficio de la acreditación. [Archivo de video]. Recuperado de https://www.youtube.com/watch?v=mDCEGTr5Qpc.

Nias, J. (1999). Teachers' Moral Pusposes: Stress, Vulnerability, and Strength. Vanderberghe y A.M. Hubermand (Eds.), Understanding and preventing teacher burnout. A sourcebook of international research and practice (pp. 223-237). Cambridge, New York.: Cambridge University Press.

Oficio SINAES-236-2006.

Palacios, J. (2015). University Students Stuck Over English. EuroWeeklyNews. Recuperado el 14 de Julio de 2015 de: http://www.euroweeklynews.com/news/costa-delsol/item/125926-university-students-stuck-overenglish?highlight=WyJlbmdsaXNoliwibGFuZ3VhZ2UiLCJlbmdsaXNolGxhbmd1YWdIII $0 \% 3 \mathrm{D}$. 
El efecto de la calidad en un programa de estudios acreditado: caso del Bachillerato en la Enseñanza del Inglés en la Universidad Nacional.

Lindsay A. Chaves Fernández, Didier Rojas Cerdas, Olga L. Chaves Carballo

Presidencia de la República. (2008-2017). Costa Rica Bilingüe. Recuperado de http://www.aliarse.org/documentos/Congreso_II/Marta\%20Blanco.pdfUniversidad Nacional. Escuela de Literatura y Ciencias del Lenguaje. 31de julio del 2001. Acta de la Asamblea de la Unidad Académica, sesión ordinaria №2-2001.

Vicerrectoría de Desarrollo. Plan Estratégico de Mediano Plazo (2013-2017). Universidad Nacional de Costa Rica. 


\title{
(Anexo 1)
}

\section{Cuestionario: Percepción Bachillerato en la Enseñanza del Inglés}

\author{
Proyecto Gestión de la Calidad del BEI \\ Escuela de Literatura y Ciencias del lenguaje \\ Universidad Nacional de Costa Rica
}

Estimados estudiantes:

Nuestra carrera continúa con el arduo proceso de mejoramiento con el propósito de valorar cuáles son sus fortalezas y determinar posibles aspectos que requieran ser mejorados. En este sentido, el presente cuestionario tiene como finalidad conocer su opinión sobre diversos aspectos relacionados con el Plan de Estudio de la Carrera del Bachillerato en la Enseñanza del Inglés.

Por favor completar el cuestionario en su totalidad. La información que usted ofrezca es confidencial.

1. ¿Considera usted que una carrera acreditada le trae beneficios para su futura profesión? Justifique su respuesta.

2. ¿Cuáles aspectos o actividades debería contener o profundizar el Plan de Estudio de manera que se ajuste más a la realidad laboral?

Por favor, marque las opciones que correspondan:

( ) Metodologías, estrategias, técnicas y tácticas para enseñar una segunda lengua

( ) Evaluación diagnóstica

( ) Paradigmas y enfoques educativos 
( ) Habilidades de expresión oral

( ) Habilidades de expresión escrita

( ) Lingüística

( ) Literatura en inglés

( ) Cultura de la lengua meta

( ) Planeamiento de unidades y lecciones

( ) Diseño y evaluación de materiales didácticos

( ) Diseño de pruebas comunicativas

( ) Diseño de procesos e instrumentos para la evaluación continua (alternativa o auténtica)

( ) Investigación educativa

( ) Evaluación de los aprendizajes

( ) Prácticas profesionales

( ) Educación inclusiva (adecuaciones curriculares)

3. ¿Cuáles son las destrezas más importantes que requiere actualmente un profesor en la enseñanza del inglés? Enumere las destrezas, siendo el 1 la destreza más importante.

( ) Competencia metodológica

( ) Competencia comunicativa

( ) Competencia lingüística

( ) Competencia discursiva

( ) Competencia afectiva

( ) Portador de valores

( ) Profesor-Investigador

( ) Creador de materiales

( ) Otros:

4. Enumere del 1 al 5 , siendo el 1 el mayor desafío, cuáles cree que son los principales retos para un profesor de inglés: 
( ) Empleo en la enseñanza

( ) Manejo de aula

( ) Utilización de las TIC en el aula

( ) Manejo de estudiantes con necesidades especiales

( ) Manejo de problemas sociales y económicos en los estudiantes

( ) Otros:

5. ¿Cuáles son los factores universitarios que han contribuido con su éxito académico? Enumere cinco posibles factores, siendo el 1 el más relevante.

( ) Profesores

( ) Materiales

( ) Infraestructura

( ) Nivel académico de los profesores

( ) Uso de las TIC

( ) Acceso a más oportunidades para hablar inglés

( ) Actividades de clase

( ) Ambiente de clase

( ) Apoyo administrativo

( ) Ayuda financiera

( ) Otros:

6. Señale tres de las principales fortalezas del plan de estudio del Bachillerato en la Enseñanza del Inglés en la Universidad Nacional.

7. Señale tres de las principales debilidades del plan de estudio del Bachillerato en la Enseñanza del Inglés en la Universidad Nacional. 
El efecto de la calidad en un programa de estudios acreditado: caso del Bachillerato en la Enseñanza del Inglés en la Universidad Nacional.

Lindsay A. Chaves Fernández, Didier Rojas Cerdas, Olga L. Chaves Carballo

8. ¿Qué aspectos siente que necesita mejorar para ejercer como profesor de inglés?

9. ¿Cómo se siente usted en cuanto a las oportunidades laborales después de graduarse?

10. ¿Cuál sería su primer objetivo a cumplir cuando sea profesor de inglés que causaría un impacto social en sus estudiantes?

¡Muchas gracias por su colaboración! 